\title{
SiC Nanowire Sponges as Electro-Pressure Sensors
}

Yu Chen ${ }^{\mathrm{a}, \mathrm{b}}$, Oluwafunmilola Ola ${ }^{\mathrm{b}}$, Hongmei Chen ${ }^{\mathrm{c}}$, Nannan Wang ${ }^{\mathrm{a}, *}$, Yongde Xia ${ }^{\mathrm{b}}$, Yanqiu Zhu ${ }^{\mathrm{b}, *}$ ${ }^{a}$ GIFT (Guangxi Institute for Fullerene Technology), School of Resources, Environment and Materials, Guangxi University, Guangxi 530004, China

${ }^{\mathrm{b}}$ College of Engineering, Mathematics and Physical Sciences, University of Exeter, Exeter EX4 $4 S B, U K$

${ }^{\mathrm{c}}$ School of Materials Science and Engineering, Jiangsu University of Science and Technology, Zhenjiang, 212003, China

Corresponding author:

*E-mail: wangnannan@gxu.edu.cn; Y.Zhu@exeter.ac.uk

\section{Abstract}

Light-weight, flexible and highly porous ceramic are very attractive to engineering applications due to their good inertness, stable and excellent mechanical properties. We here report such SiC nanowire (SiCNW) sponges and demonstrate their multi-functionalities. They were simply generated by reacting $\mathrm{SiO}_{2}$ with the sustainable kitchen sugar, using $\mathrm{NH}_{4} \mathrm{Cl}$ as a blowing agent. The as-grown highly porous SiCNW sponges exhibit a core-shell structure, with an extremely low density in the range of $115-125 \mathrm{mg} / \mathrm{cm}^{3}$ (against $3.21 \mathrm{~g} / \mathrm{cm}^{3}$ for the bulk). The core part is comprised of short and tangled SiC whiskers with $\mathrm{SiC}$ flakes embedded, whilst the shell layer consists of numerous smooth SiCNWs of hundred micrometres long. These sponges exhibit a compressive modulus of $\sim 1.35 \mathrm{MPa}$ and recoverability under cyclic compression loading for 100 cycles at a strain of $20 \%$. Meanwhile, the SiCNW sponges exhibit interesting electromechanical sensing capability with a gauge factor up to 87 and stable wide-range compression-resistance responses that are hundreds of times better than those of carbon-based composite sensors. Furthermore, the high porosity $(96.1 \%-96.4 \%)$ of sponges gives rise to a very low thermal conductivity of merely $1.01 \mathrm{~W} / \mathrm{mK}$ at room temperature, demonstrating their excellent thermal insulation potential. These light-weight, highly porous, thermally insulating features of the SiCNW sponges can be further exploited in electromechanical micro-devices for monitoring structural damage or capturing impacts, at high temperature environment.

\section{Keywords}

Carbothermal reduction, $\mathrm{SiC}$ nanowire, $\mathrm{SiC}$ sponge, Pressure sensor, Thermal insulation 


\section{Introduction}

Recently, three dimensional nanostructures with electromechanical sensing capabilities have been exploited as piezoresistive sensors for pressure detection, ${ }^{1}$ vibration and noise monitoring, ${ }^{2}$ human motion capturing, ${ }^{3}$ and interactive wearable devices. ${ }^{4}$ Typically, the resistive pressure-sensors show advanced features of huge simplicity and high energy-efficiency. ${ }^{5}$ Composites pressure sensors made from soft polymers with a conductive filler phase such as graphene or carbon nanotubes have been intensively investigated, however the aggregation issue of these carbon materials due to their high aspect ratios results in a series of drawbacks including non-linearity, hysteresis effect, and the drift of resistance value that caused by unrecoverable plastic deformation. ${ }^{6,7,8}$ Another shortcoming is that the limited stiffness of the carbon-polymer composites significantly narrows its dynamic sensing ranges, which made them suitable only for very small loads detection. ${ }^{9}$ Moreover, their instability at high temperatures suppresses their utilisations for demanding conditions. Metallic sensors that are commonly used in structural strain monitoring suffer from limited measuring strains of less than $2 \%$, despite their appreciable good linearity and sensitivity. ${ }^{6}$

In this context, light-weight and resilient porous ceramic sponges that consist of numerous tangled ceramic nanofibers are considered as an ideal alternative since they possess competent mechanical strength, resistance to brittleness, and certain degree of compressive recoverability. The elasticitydependant electrical conductivity of $\mathrm{TiO}_{2}$ nanofibrous sponges during repeated compression has been reported. ${ }^{10}$ Nanostructured silicon carbide $(\mathrm{SiC})$ with large specific surface area, ${ }^{11}$ good thermal stability, strong chemical inertness, ${ }^{12}$ and superior thermal insulation performance compared with its dense and bulk counterpart, ${ }^{13,14}$ has been considered as a promising candidate for sensing components in microelectromechanical systems (MEMS). ${ }^{15}$

As one of the simplest and most cost-effective approaches, in-situ carbothermal reduction has been an effective technique for $\mathrm{SiC}$ nanomaterials synthesis. ${ }^{16}$ Based on the low-cost silicon oxide $\left(\mathrm{SiO}_{2}\right.$, $\mathrm{SiO}$, or $\mathrm{SiOC}$ ), various forms of porous carbon template (graphene foam $(\mathrm{GF}),{ }^{17,18}$ carbonised dough ${ }^{13}$ ) were used to create porous $\mathrm{SiC}$ via carbonisation. ${ }^{12}$ The resultant structures strongly depend on the initial $\mathrm{C} / \mathrm{SiO}_{2}$ matrix and interaction between reagents. However the control of $\mathrm{SiC}$ crystal growth and the formation of porous structure at high temperatures remain challenging, since improper product structures could lead to poor mechanical properties. ${ }^{19,20}$ To achieve SiCNW sponges with better shape stability, efforts have been focused on the growth of SiC nanowires on substrates such as $\mathrm{C} / \mathrm{C}$ composites, ${ }^{21}$ and macroscopic $\mathrm{SiC}$ host. ${ }^{22}$ Electrospinning has emerged as a frequently used technique for producing $\mathrm{SiO}_{2}$-containing polymer nanowires, which can be used as starting materials

in the carbothermal reaction for $\mathrm{SiC}$ nanowire formation. ${ }^{23,24}$ Wang et al. synthesised well-aligned 
SiC nanofibers through carbothermal reaction of silicon and electrospun PAN precursor. ${ }^{25}$ However, electrospun fibres are generally created in the form of a close-packed membrane so they cannot easily be transformed to 3D structures, thereby restricted their wide applications. ${ }^{10}$ To overcome this, additional freeze-casting technique was required to assemble them into $3 \mathrm{D}$ aerogels. ${ }^{26}$ Hence, a simple and efficient approach for the development of SiCNW sponges is needed.

Inspired by a facile template/catalyst-free sugar-blowing technique, ${ }^{27,28}$ which created the 3D strutted graphene (SG), this study demonstrates the generation of SiCNW sponges via a one-step in-situ template/catalyst-free carbothermal reaction using $\mathrm{SiO}_{2}$ and sugar-derived carbon. The microstructure, mechanical properties, and thermal conductivity of the as-obtained SiCNW sponges will be investigated comprehensively, and the pressure-dependent electrical responses will be illustrated for the first time, which makes an original contribution to utilising SiCNW sponges as a pressure sensor.

\section{Experimental methods}

\section{SiCNW sponge fabrication}

The $\mathrm{NH}_{4} \mathrm{Cl}$ porogen (Sigma-Aldrich, UK, $\geq 99.5 \%$ purity,) was first mixed with sugar (kitchen-use sugar from Whitworths Co.) at a weight ratio of 1:1. The granular $\mathrm{NH}_{4} \mathrm{Cl} /$ sugar mixture were grounded into fine powders before being blended with $\mathrm{SiO}_{2}$ powder (approx. 99\%, particle size: 0.5$10 \mu \mathrm{m}$, Sigma-Aldrich) at different weight ratios, and the mixed powders were then subjected to a pressure at 5 bar using a mould with a dimension of $12.5 \times 25 \times 4.5 \mathrm{~mm}^{2}$, to prepare the green bodies. This green body was finally treated at $1500{ }^{\circ} \mathrm{C}$, at a ramping rate of $5{ }^{\circ} \mathrm{C} / \mathrm{min}$ and a dwell time of $4 \mathrm{~h}$ in $\mathrm{Ar}$ atmosphere (flow rate $70 \mathrm{ml} \mathrm{min}^{-1}$ ). To obtain pure $\mathrm{SiC}$ sponges from the as-synthesised $\mathrm{SiC} / \mathrm{C}$ composites, a further thermal treatment at $800{ }^{\circ} \mathrm{C}$ for $2 \mathrm{~h}$ in air was performed to remove the superfluous carbon via oxidation.

\section{Characterisation}

The XRD powder diffraction patterns were recorded on a Bruker D8 Advance diffractometer with a $\mathrm{Cu} \mathrm{K \alpha}$ radiation $(\lambda=0.154 \mathrm{~nm}$, operated at $40 \mathrm{kV}$ and $40 \mathrm{~mA})$, at a time interval of $1 \mathrm{~s}$ and a step size of $0.02^{\circ}$, within the $2 \theta$ range from $10^{\circ}$ to $80^{\circ}$. ICDD cards No. 01-089-2352 and 01-075-0254 were used for the identification of $\alpha-\mathrm{SiC}$ and $\beta-\mathrm{SiC}$, respectively. Their weight percentage was determined via the Rietveld refinement method. SEM observations were performed using a Hitachi S3200N SEM-EDS machine. TEM investigations were carried out using a JEM-2100 TEM, operated at $200 \mathrm{kV}$. For the TEM sample preparation, the crushed SiC samples were ultrasonically dispersed in ethanol for $10 \mathrm{~min}$ and the suspension was then dropped onto a holey carbon coated copper grid 
(300 mesh, Agar). Thermal gravimetric analysis was conducted using a TA SDT Q600 TGA-DSC instrument, at a ramping rate of $10{ }^{\circ} \mathrm{C} \mathrm{min}^{-1}$ under an air environment. Fourier-transform infrared (FTIR) spectroscopy was conducted by means of a Tensor-27 FTIR spectrometer in the wavenumber range of $2000-500 \mathrm{~cm}^{-1}$.

The cyclic compression test of SiC nanowire sponges at a pre-set strain of $20 \%$ was carried out on a Lloyds EZ20 advanced universal mechanical testing system, using a $500 \mathrm{~N}$ detection cell at a loading rate of $8 \mathrm{~mm} \mathrm{~min}^{-1}$.

The pressure-dependent electrical response was analyzed by KEITHLEY 2612B System Sourcemeter Instruments that connected with an Instron ElectroPlus E10000 All-Electric Dynamic Test Instrument and a computer for data collection. The sample was placed between two parallel copper plate electrodes. The dynamic resistance responses of the sensor system were examined by applying a cyclic compressive load on the sample at $1 \mathrm{~Hz}$ between $50 \mathrm{~N}$ and $250 \mathrm{~N}$ for $400 \mathrm{~s}$.

Thermal diffusivity evaluations of the nanocomposites were conducted on a NETZSCH LFA 467 HyperFlash machine. Prior to testing, the $1 \mathrm{~cm}^{2}$ samples were spray-coated with a thin layer of graphite, to improve the signal to noise ratio and enhance the light emission/absorbance during analyses. The thermal conductivity $(\lambda)$ was extrapolated from the dataset obtained from thermal diffusivities $(\alpha)$, using $\lambda=\alpha \rho C p$, where $\rho$ and $C$ p represent the density and specific heat capacity of the sample, separately. An average value was obtained from the software after 3 runs.

\section{Results and Discussion}

\section{Fabrication of SiCNW sponges}

The starting materials containing sugar and $\mathrm{SiO}_{2}$ with different weight ratios $(1.25: 1,1.5: 1,1.75: 1$, 2:1) were mechanically mixed, and $\mathrm{NH}_{4} \mathrm{Cl}$ with the same weight of sugar was added as the porogen. The mixture was pressed into rectangular blocks of $12.5 \times 25 \mathrm{~mm}^{2}$ prior to sintering during which the in-situ carbothermal reaction took place. Our prerequisite results based on the XRD analyses (Figure S1) showed that a sugar/ $\mathrm{SiO}_{2}$ weight ratio of at least 1.75:1 was enough for the depletion of $\mathrm{SiO}_{2}$ powders. As shown in Figure 1, the compacted green body led to little shrinkage and good morphology control over the final products. The bulk densities of resultant light-green SiCNW sponges range from $115 \mathrm{mg} \mathrm{cm}^{-3}$ to $125 \mathrm{mg} \mathrm{cm}^{-3}$, and the theoretical porosity is estimated using the following equation:

$$
\mathrm{Pt}=\left(1-\frac{\mathrm{Pb}}{\mathrm{Pd}}\right) \times 100 \%
$$


Where the $\mathrm{Pt}, \mathrm{Pb}$, and $\mathrm{Pd}$ represent the porosity, bulk density, and particle density, respectively. The calculated porosity of the foams is $96.1 \%-96.4 \%$.

During sintering, with the increase of temperature at a controlled rate of $5{ }^{\circ} \mathrm{C} \mathrm{min}^{-1}$, the molten sugar was gradually polymerised to melanoidin at around $250^{\circ} \mathrm{C}$. The melanoidin then underwent a series of deoxidation, dehydrogenation, solid-phase carbonisation, and multi-phase graphitisation at high temperature, before resulting in the final carbon product. ${ }^{27}$ Meanwhile, a porous structure was simultaneously created by the gaseous $\mathrm{HCl}$ and $\mathrm{NH}_{3}$ released from decomposing of $\mathrm{NH}_{4} \mathrm{Cl}$ :

$$
\mathrm{NH}_{4} \mathrm{Cl}(\mathrm{s}) \rightarrow \mathrm{HCl}(\mathrm{g})+\mathrm{NH}_{3}(\mathrm{~g})
$$

The abundant active sites within the sponge led to further carbothermal reaction between $\mathrm{C}$ and $\mathrm{SiO}_{2}$ :

$$
\mathrm{SiO}_{2}(\mathrm{~s})+3 \mathrm{C}(\mathrm{s}) \rightarrow \mathrm{SiC}(\mathrm{s})+2 \mathrm{CO}(\mathrm{g})
$$

To determine the heating temperature for the superfluous carbon elimination treatment, TGA under air environment was conducted between $20-1000{ }^{\circ} \mathrm{C}$. The $\mathrm{SiC} / \mathrm{C}$ composite shows a $24.4 \%$ weight loss from $530{ }^{\circ} \mathrm{C}$ to $750{ }^{\circ} \mathrm{C}$, which corresponds to loss of $\mathrm{CO}_{2}$ due to the reaction of $\mathrm{C}$ and $\mathrm{O}_{2}$ and converts to a $\mathrm{SiC}$ content of $75.6 \%$ within the composites, as shown in Figure 2a. A $1.5 \%$ weight increase from $750{ }^{\circ} \mathrm{C}$ to $1000{ }^{\circ} \mathrm{C}$ is due to the oxidation of $\mathrm{SiC}$ after the carbon content was fully burnt off (oxidised). Based on these results, an additional thermal treatment at $800{ }^{\circ} \mathrm{C}$ for $2 \mathrm{~h}$ in air was designed to remove the excessive carbon residue in the sponge.

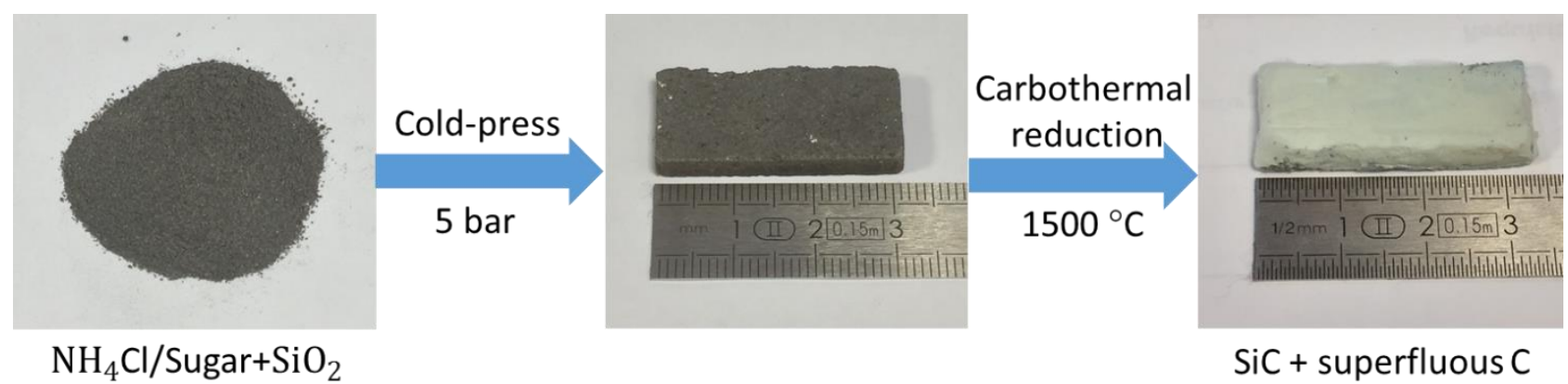

Figure 1. Digital photos of the raw powder mixture, green body and sintered sponge obtained at 1500 ${ }^{\circ} \mathrm{C}$ for $240 \mathrm{~min}$, showing the well-preserved geometry after sintering.

\section{Morphological and structural characterisation}

Figure $2 \mathrm{~b}$ shows the XRD results of the as-obtained samples before and after the carbon removal treatment. Peaks at $35.7^{\circ}, 41.4^{\circ}, 60^{\circ}, 71.8^{\circ}$ and $75.6^{\circ}$ correspond to the (111), (200), (220), (311) and (222) planes of $\beta$-SiC, whilst the peak at around $33.6^{\circ}$ indicates the coexistence of hexagonal phases 
$(\alpha-\mathrm{SiC}){ }^{29}$ A weight percentage between the two polytypes is determined to be $64.5: 35.5$ by the Rietweld refinement method (Figure S2). Meanwhile, the disappearance of the broad peak at around $14.8^{\circ}$ for the pure $\mathrm{SiC}$ sponge shows the successful elimination of amorphous carbon compounds. No other crystalline peaks are detected.

Carbothermal reaction hugely depends on the nature of the starting materials, along with the extent of $\mathrm{SiO}_{2}-\mathrm{C}$ interactions and the temperature regime. ${ }^{12}$ Higher $\mathrm{C}$ content over $\mathrm{Si}$ is energetically more favourable, since more carbon could accelerate the carbonisation of free Si and suppress the formation of free Si. The XRD result together with TGA analysis indicate that an adequate composition of the starting material and thermal treatment conditions for the formation of $\mathrm{SiC}$ have been obtained.
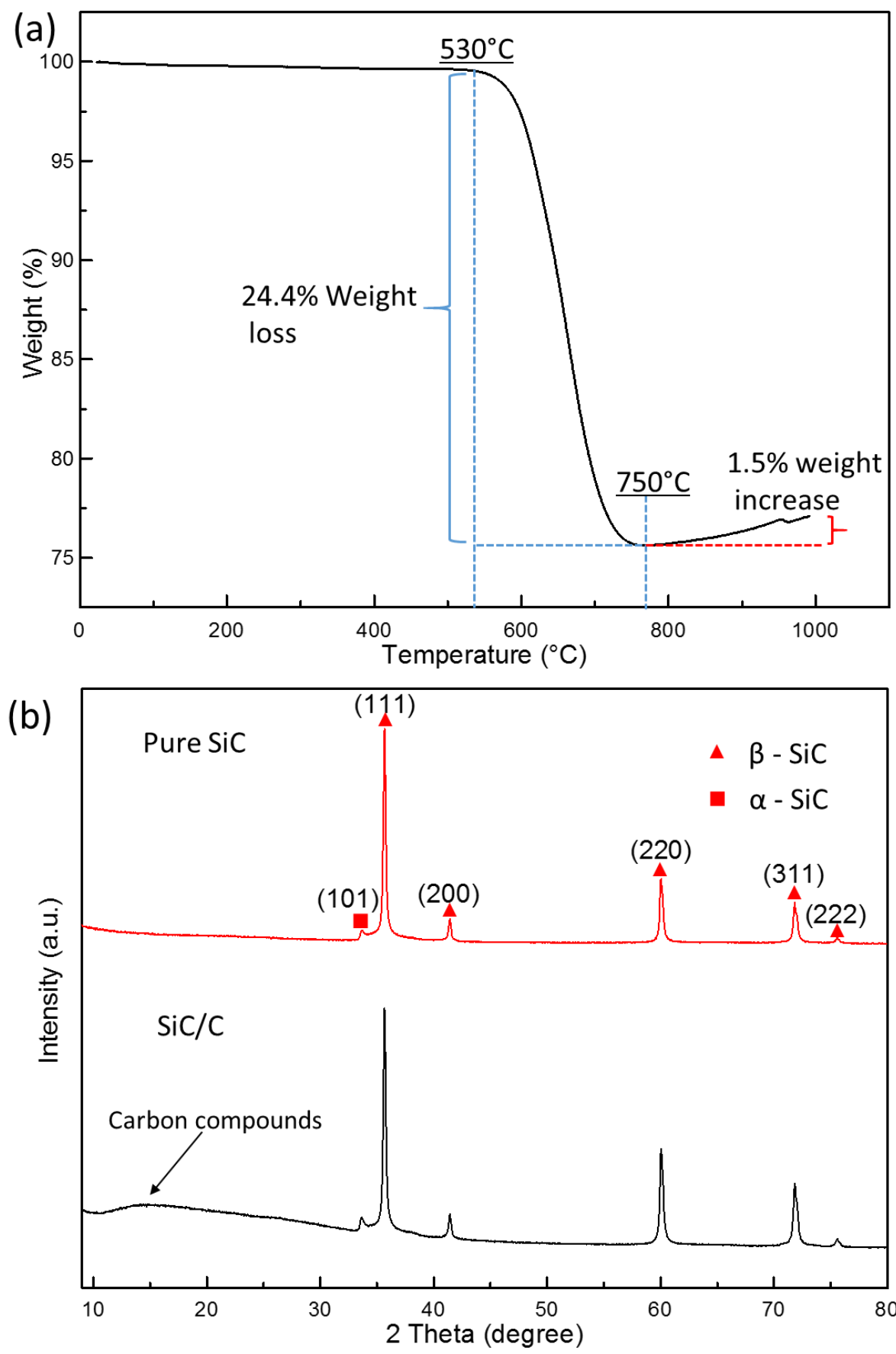
Figure 2. (a) TGA result of the as-obtained $\mathrm{SiC} / \mathrm{C}$ before the carbon removal treatment. (b) XRD profiles of the as-obtained $\mathrm{SiC} / \mathrm{C}$ and purified $\mathrm{SiC}$ sponges after the carbon removal at $800{ }^{\circ} \mathrm{C}$.

From the fracture surface of SiCNW sponges shown in Figure 3a-d, a rough core-shell feature is visible. The core part is comprised of interconnected and tangled SiC whiskers with a small number of flakes. The whiskers are a few hundred $\mathrm{nm}$ to tens of $\mu \mathrm{m}$ in length, and the flakes are up to $\mu \mathrm{m}$ in size. These shorter whiskers in the core is owing to the pre-applied compact pressure which gives rise to relatively closed porous structure, restricting the free movement of gaseous $\mathrm{SiO}$ and $\mathrm{CO}$, and as a result hinders the growth of SiCNWs. By contrast, owing to the exposure to the flowing Ar, a continuous supply and transport of Si- and C- containing gaseous reactants at the external surface of the greenbody was realised, so that SiCNWs of $\sim 100-200 \mu \mathrm{m}$ long constituting a shell layer with a thickness of $\sim 160-220 \mu \mathrm{m}$ were generated. This evidently displays the principle of catalyst-free vapor-solid (VS) mechanism, where the morphology of nanowires is largely dictated by the interaction of gaseous reactants.

TEM analyses confirm that the morphologies of nanofibers from the shell and core parts are similar (inset of Figure $3 \mathrm{c}$ and d). They exhibit diameters ranging from $20 \mathrm{~nm}$ to $200 \mathrm{~nm}$, with majority in the range of 50-80 $\mathrm{nm}$. Further EDS analyses (inset of Figures $3 \mathrm{c}$ and d) verify that these nanowires contain $\mathrm{Si}$ and $\mathrm{C}$, with a trace amount of $\mathrm{O}$ that is inevitable due to $\mathrm{SiO}_{2}$ always forming a thin layer on the $\mathrm{SiC}$ surface as a by-product. The content of $\mathrm{O}$ element in the nanowires from the shell is higher than that of the core counterpart, which is opposite to the distribution of the $\mathrm{C}$ element, suggesting the shell functions as the oxidation resistant layer. High resolution TEM observations (Figures 3e and f), together with selected area electron diffraction (SAED, Figure 3e, inset), have revealed the stacking faults along the (111) direction of the SiC nanowire, which agrees well with the XRD analysis that the $\mathrm{SiC}$ is dominantly $\beta$ phase. 

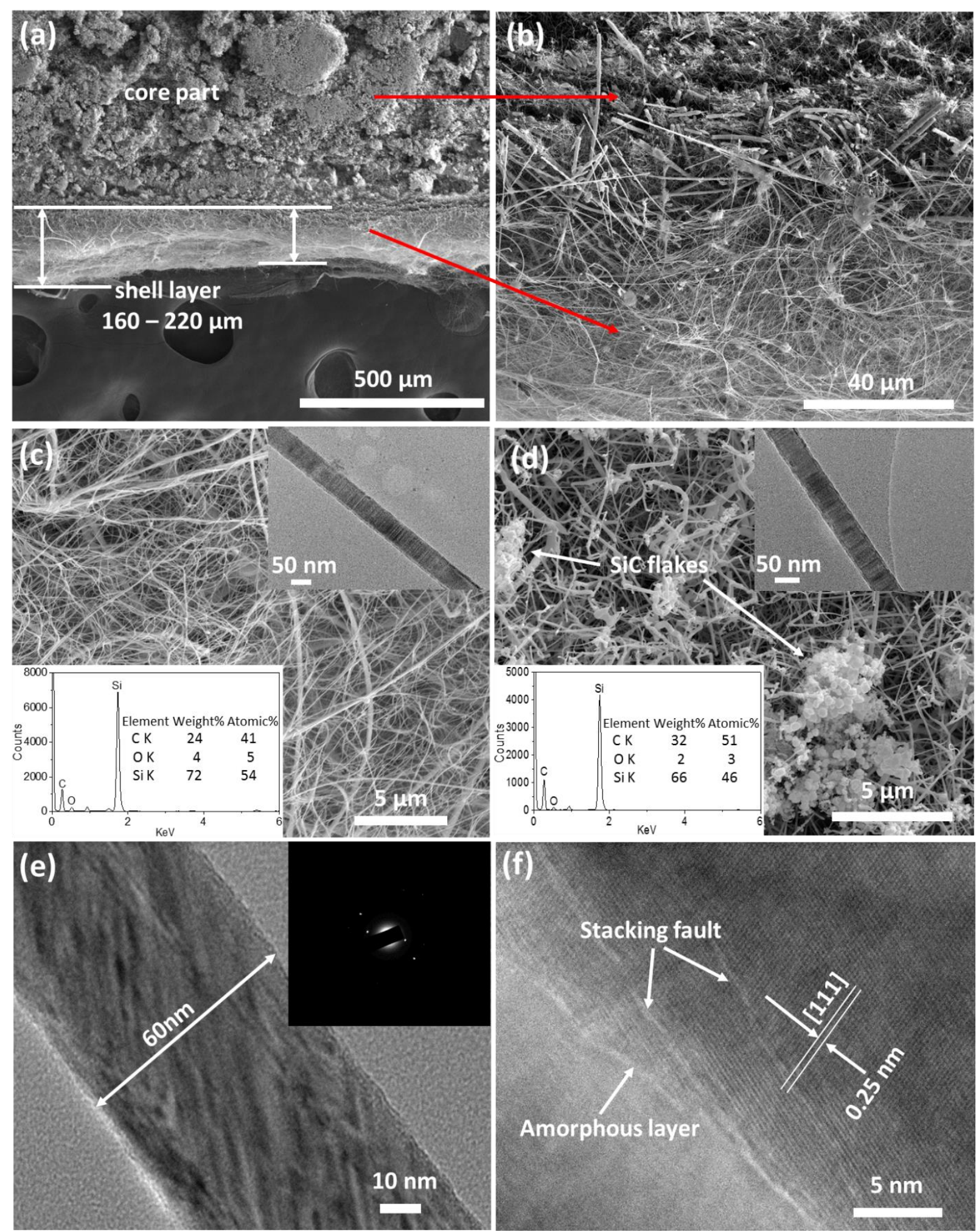

Figure 3 ( $a, b)$ SEM images of the core-shell feature of the sponge. (c, d) SEM images of the nanowires from the shell and core regions, respectively, and the inset showing TEM images of individual nanowires and their corresponding TEM - EDS analyses. (e, f) High magnification TEM images of a nanowire. Inset in image (e) is its SAED pattern. 
These long and smooth SiC nanowires are evidently generated by the catalyst-free Vapor-Solid (VS) mechanism, which is associated with the reaction between Si- and C-containing gaseous reactants in multiple steps. Firstly, $\mathrm{SiO}$ gas is generated on the interface between $\mathrm{SiO}_{2}$ and carbon source via following reactions: ${ }^{30}$

$$
\begin{gathered}
\mathrm{SiO}_{2}(\mathrm{~s}, \mathrm{l})+\mathrm{C}(\mathrm{s}) \rightarrow \mathrm{SiO}(\mathrm{g})+\mathrm{CO}(\mathrm{g}) \\
\mathrm{SiO}_{2}(\mathrm{~s}, \mathrm{l})+\mathrm{CO}(\mathrm{g}) \rightarrow \mathrm{SiO}(\mathrm{g})+\mathrm{CO}_{2}(\mathrm{~g})
\end{gathered}
$$

Subsequently, $\mathrm{SiO}$ gas reacts with active gaseous carbon source and generates $\mathrm{SiC}$ nanowire nucleus, which grow into nanowires by a continuous supply of $\mathrm{SiO}$ and $\mathrm{CO}$ gas, the mass transport of $\mathrm{C}$ is also facilitated by the flow of Ar gas: ${ }^{31}$

$$
\mathrm{SiO}(\mathrm{g})+3 \mathrm{CO}(\mathrm{g}) \rightarrow \mathrm{SiC}(\mathrm{s})+2 \mathrm{CO}_{2}(\mathrm{~g})
$$

\section{Mechanical properties}

Uniaxial compression test was carried out to evaluate the compressive behavior of the sponges. As shown in Figure $4 \mathrm{a}$, under $\sim 10 \%$ strain, the low mechanical response with stress up to $127.7 \mathrm{kPa}$ suggests that fluffy shell layer with thickness in the range of $\sim 160-220 \mu \mathrm{m}$ was first responded to the compression load. Three other regions are subsequently observed in the stress-strain graph: an elastic region, a short plateau region, and a densification region. From the linear elastic region, an estimated compressive modulus of $\sim 1.35 \mathrm{MPa}$ was obtained. It is worth noting that the compressive brittle failure manner that usually observed in ceramic materials is absent in this context. As illustrated in Figure $4 \mathrm{~b}$ and $\mathrm{c}$, the long fibres in the shell must have experienced serious densification during the test. Surprisingly, the morphological integrity of the sponge is well-preserved, without macroscopic fractures or cracks on the surface even at a $70 \%$ strain. This is probably accredited to the robustness of whiskers in the core part, which helped to maintain the initial morphology of the sponge. It is believed that the co-existence of $\mathrm{SiC}$ flakes also played a role in providing the good mechanical strength and structural integrity. The excellent compressive responses demonstrated by the SiCNW sponges have shown that the major drawbacks of brittleness and high flaw sensitivity known to traditional porous ceramic sponges have been overcome.

The SiCNW sponges endured 100 loading-unloading compression cycles at a strain of $\varepsilon=20 \%$, and exhibited certain degree of recoverability, as displayed in Figure 4d. Post-testing SEM examinations of the sponge (Figures $4 \mathrm{e}$ and $\mathrm{f}$ ) showed that only the shell layer underwent irreversible deformation/condensation, and an $87 \%$ recovery to the original height of the sponge was achieved 
after the first cycle which corresponds to a residue strain of $13 \%$. However, the compressive response of the sponges was then stabilised after the first cycle, and they exhibited a 16\% residual strain (from $13 \%$ ) and $68 \%$ of its original maximum stress even after 100 loading-unloading cycles, respectively. Further SEM examinations revealed that nanofibers in the shell layer were much denser than those of prior to testing, whist the nanofibers in the core part of the sponge appeared to have experienced negligible condensation and deformation (Figure 4f). Therefore, the discrete plastic deformation of the randomly tangled nanofibers in the shell layer is believed to have absorbed the majority of the sponge deformation during the cyclic compression testing, being the leading cause of the stress drop. The nanofibers in the core part underwent only an elastic deformation and were almost fully recovered after the load was released, as schematically presented in Figure $4 \mathrm{~g}$.
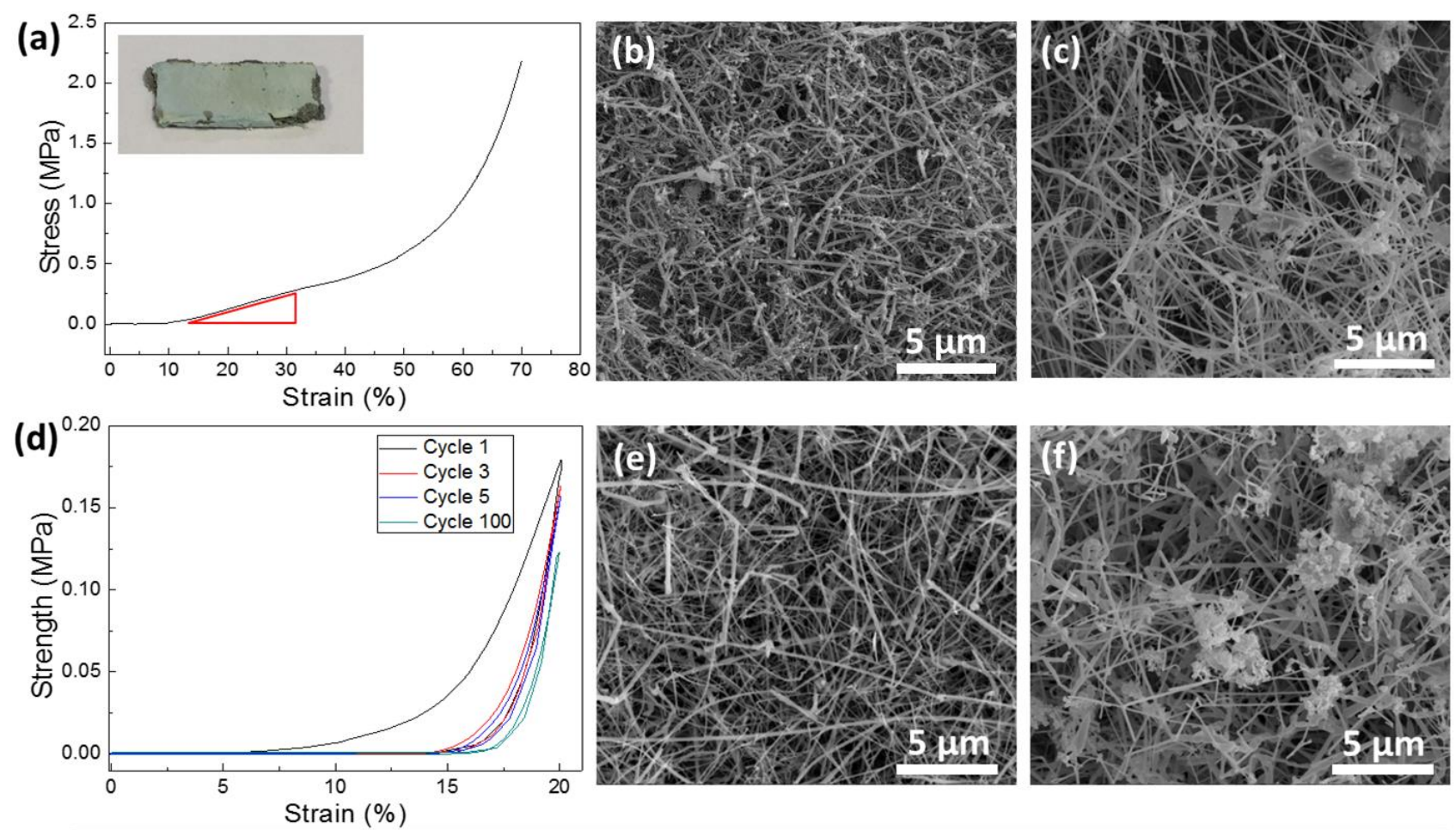

(g)

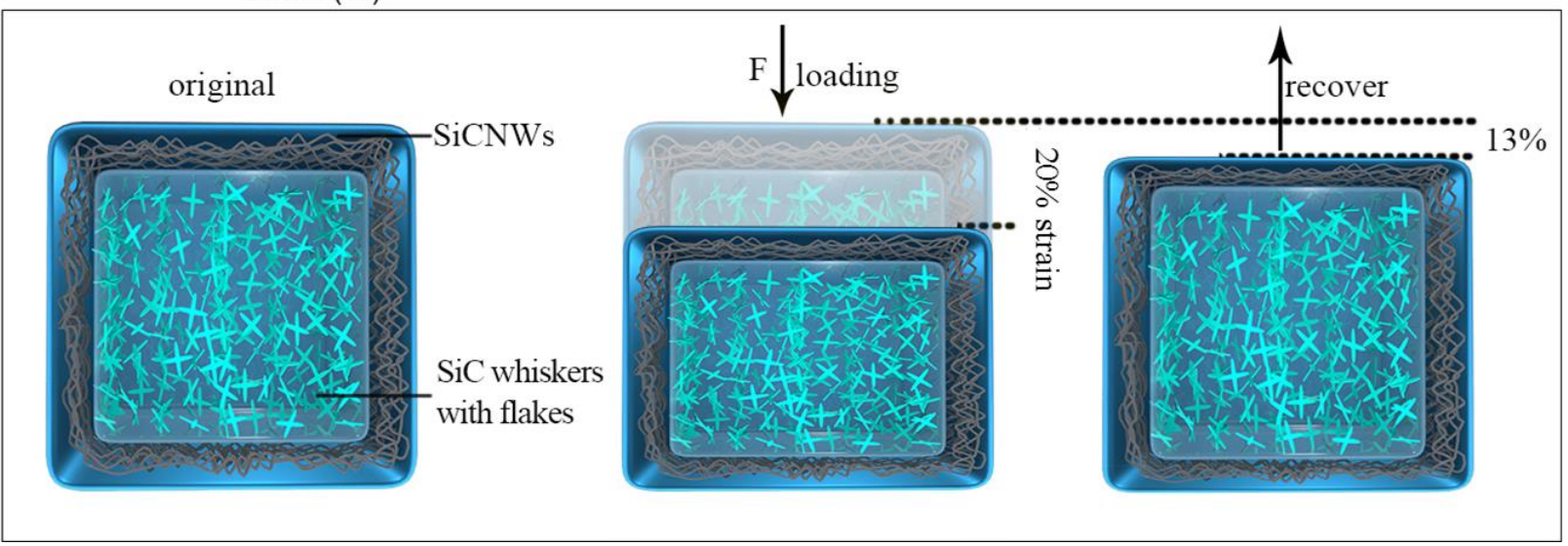

Figure 4. (a) Compressive stress-strain curves of the SiCNW sponges. The inset of post-testing sample shows no noticeable cracks. (b and c) SEM images of the post-compression samples, with (b) 
showing the overall shell part and (c) showing the core part. (d) Cyclic compressive stress-strain curves of the SiCNW sponges under a fixed strain of 20\%. (e and f) SEM images of the postcompression samples, of which (e) showing the overall shell part, and (f) presenting the core part. (g) Illustrating the different responses of the shell and core of the sponges during the compression test.

To clarify the detailed cyclic compressive responses of the core part, we carefully peeled off the outer layer and then carried out the compression test under the same conditions. We found that the inner sponge was considerably more responsive and reacted more in the first cycle, as shown in Figure 5a. A yield point was observed on the stress-strain curve. Considering the SEM image shown in Figure $5 \mathrm{~b}$, we believed that this yield point might correspond to the condensation of the SiC fibres and flakes in the core part. In subsequent cycles, the core part exhibited less recoverability, possibly due to the permanent deformation via knotting of some whiskers (Figure $5 \mathrm{~b}$ inset) ${ }^{10}$ however, a new mechanical equilibrium was established.
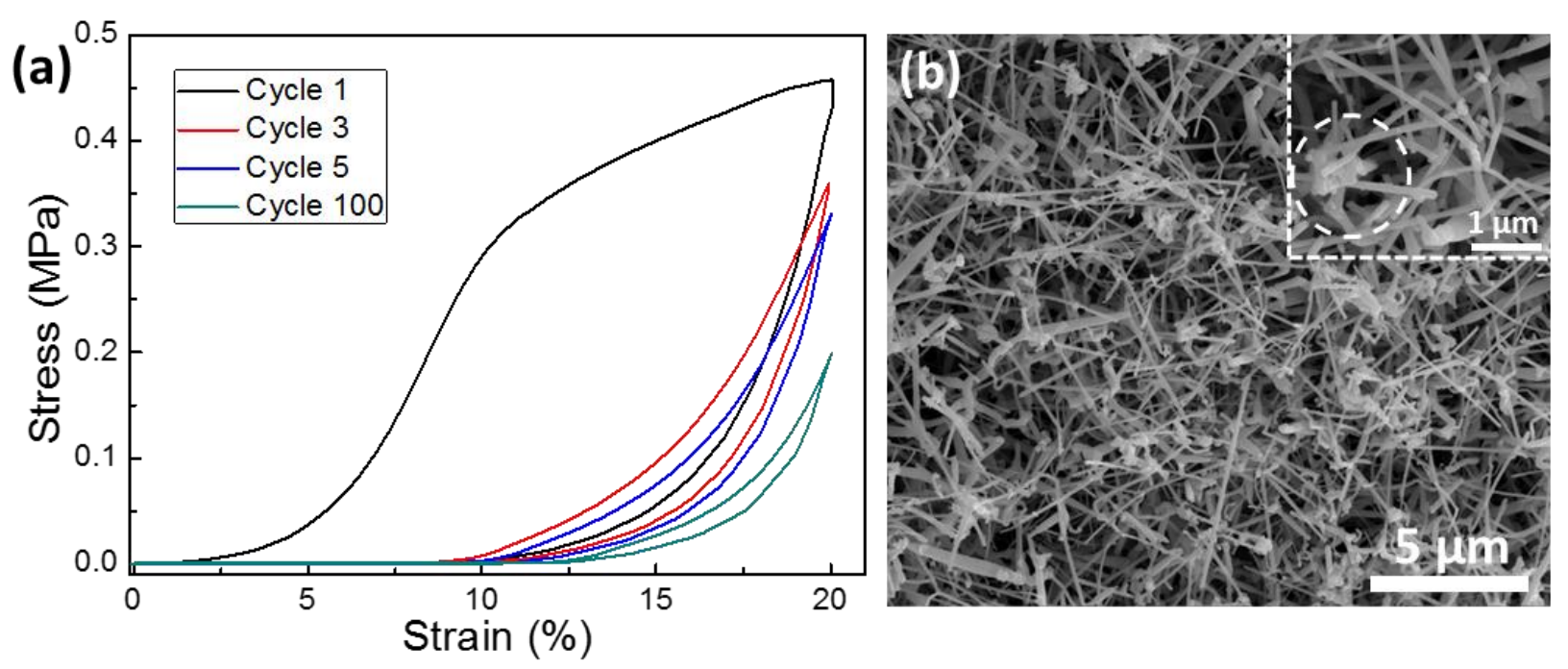

Figure 5. (a) Compressive stress-strain curves of the core part, and (b) SEM image of the post-test sample. Inset is a higher magnification image, and the dashed circle showing a knot assembled by the fibres.

All the above results suggest that the shell layer of the SiCNW sponges served as a protective layer that enabled the sponge to have compressive energy absorbing capabilities in the low strain regions $(0-\sim 10 \%)$, but the whiskers and flakes in the core part provided the mechanical strength and compressive recoverability for the sponge. 


\section{Pressure-dependent electrical response}

The electromechanical properties of the $\mathrm{SiCNW}$ sponges were examined by measuring the changes of resistance versus the cyclic load applied, and the results are displayed in Figure 6. As illustrated in Figure 6a and b, the electrical resistance decreased from $1007 \Omega$ to $477 \Omega$ with the rise of applied load from $50 \mathrm{~N}$ to $250 \mathrm{~N}$ (equals to the pressure from $\sim 130.2$ to $\sim 651 \mathrm{kPa}$ ), but completely recovered upon unloading. Meanwhile, the sponge exhibited abnormally high sensitivity, with a strain gauge factor (GF) up to 87.27 at the very beginning of the test with strain change of no more than $1 \%$, which dropped to 1.63 in the high strain range of 20.5-23.35\%. During the loading-unloading process, numerous nanowires under compression would slide and bend which made them touching the neighbour nanowires. As a result, numerous temporary knot contacts would be created which acted as shortened conduction paths throughout the sample. Upon unloading, these temporary contacts would disappear, hence the resistance returned completely to its initial value.

Given the excellent cyclic compression behaviour of the sponge with $20 \%$ strain, as presented earlier, it is interesting to investigate the dynamic resistance responses of the sensor system under cyclic compression. Figures $6 \mathrm{c}$ shows the interception of harmonic electrical resistance variations within cycles of 1-20 and 380-400. The SiCNW sponges exhibited superb sensing repeatability, with negligible decays even after 400 loading-unloading cycles. To explicitly compare the cyclic sensing performance of pure SiCNW sponges with composites sponges, the behaviour of the $\mathrm{SiC} / \mathrm{C}$ nanowire sponge (without the elimination of carbon) was also assessed as a reference. The result (Figure S7) showed that the composite sponge exhibited much lower resistance, however, just like polymerMWNTs composites sensors that suffers drift almost in every cycle, ${ }^{6}$ they experienced pronounced drift during dynamic test, losing over $50 \%$ of its output resistance after 400 cycles. The low resistance is due to the existence of carbon that has higher electrical conductivity than SiC, however the signal drifting should also originate from the aggregation of the residue carbon. This complementary experiment confirmed that the purified SiCNW sponges offer higher sensitivity and greater reproducibility than that containing carbon residue for electric pressure sensor applications. This finding unambiguously suggests that the single-phase sponges better off composites-based sensors in terms of both the structural and electrical stabilities. 

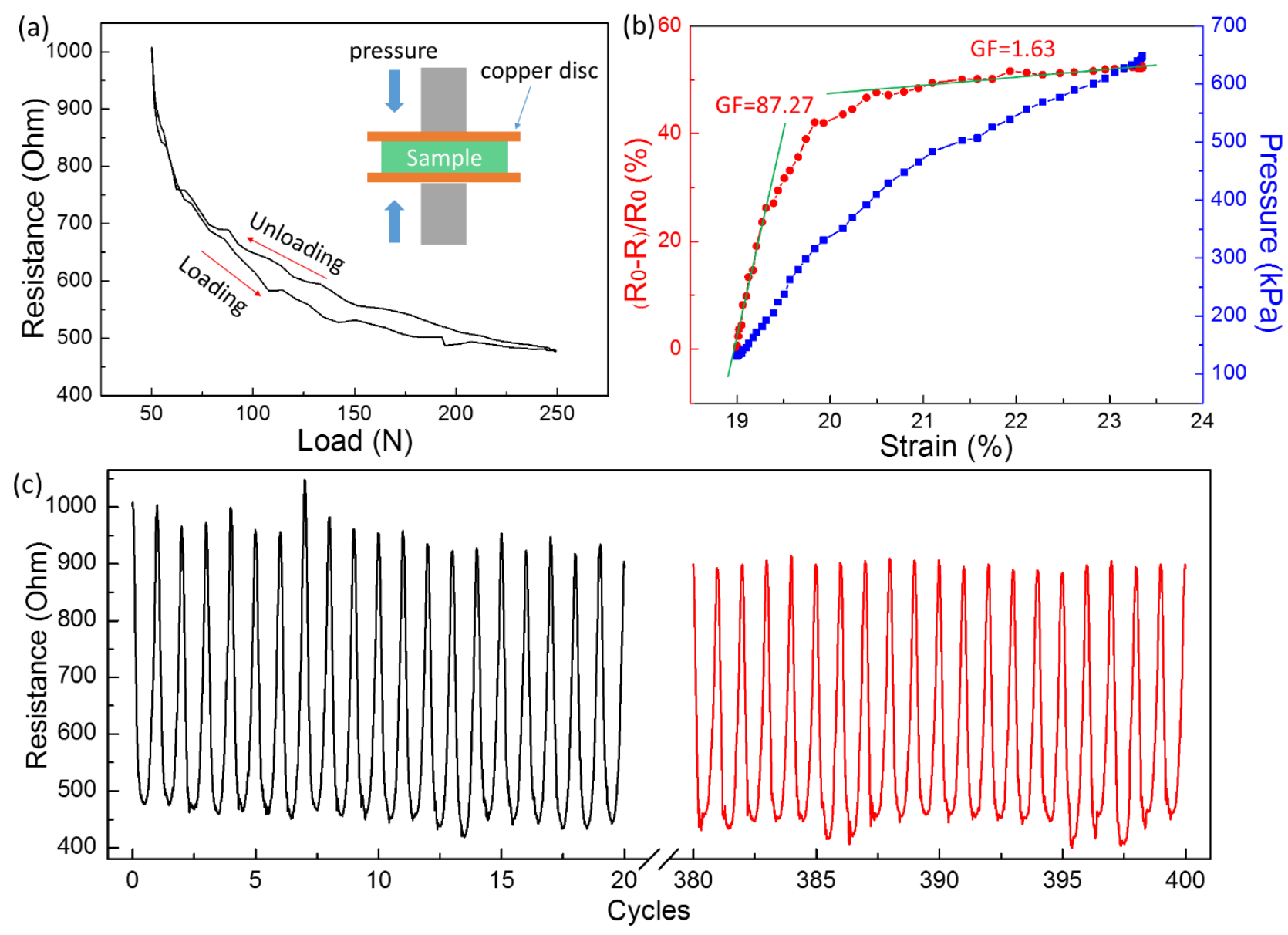

Figure 6. (a) Electrical resistance as a function of applied compressive strength. Inset shows the schematic setup for the pressure-dependent electrical response measurement. (b) Variation of electrical resistance change and applied pressure with compressing cycles. Gauge factors were derived from linear fitting. (c) Electrical resistance of SiCNW sponges at different compressing cycles, up to 400 cycles measured.

\section{Thermal insulation performance}

Compared with the intrinsic thermal conductivity of dense $\mathrm{SiC}(490 \mathrm{~W} / \mathrm{mK}),{ }^{32}$ the SiCNW sponge gives a rather low thermal conductivity ranging from $0.085 \mathrm{Wm}^{-1} \mathrm{~K}^{-1}$ to $0.13 \mathrm{Wm}^{-1} \mathrm{~K}^{-1}$ from $-50{ }^{\circ} \mathrm{C}$ $200{ }^{\circ} \mathrm{C}$ (Figure 7). As conduction is dominant heat transfer mechanism in porous media, this densityrelated property is largely dictated by the porosity since the pores act as phonon transport barriers that strongly affect the phonon mean-free-path. With the escalation of the test temperature, the higher phonons vibration velocity together with the increased phonons collision frequency give rise to the drop of the phonon mean-free-path, leading to the decline of thermal conductivity. ${ }^{33}$ An interesting relationship between porosity and thermal conductivity has been proposed, i.e. higher porosity corresponds to low thermal conductivity which is understandable, as summarised in Table 1 . The 
thermal conductivity of the SiCNW sponge is even lower than other porous SiC ceramics that were reported recently with similar porosities. This could be accredited to the relatively small diameters of the SiCNWs, suggesting that the present SiCNW sponges could act as excellent thermal insulators.

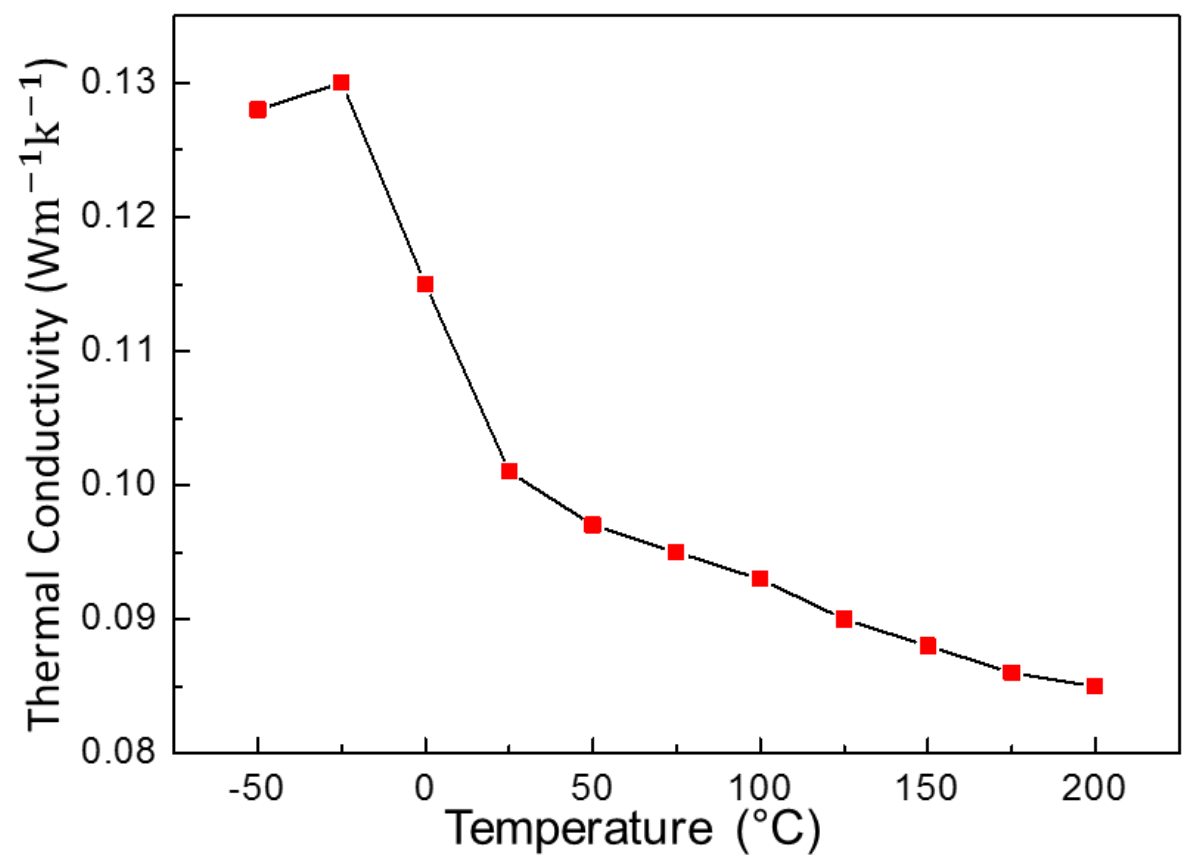

Figure 7. Thermal conductivity of the purified SiC sponge.

Table 1. Thermal conductivity of SiC sponges/foams of different porosities.

\begin{tabular}{ccc}
\hline Porosity (\%) & $\begin{array}{l}\text { Thermal conductivity at } \\
\text { room temperature }\left(\mathrm{Wm}^{-}\right.\end{array}$ & References \\
& $\left.{ }^{1} \mathrm{~K}^{-1}\right)$ & \\
\hline 40 & 19 & 34 \\
70 & 2 & 35 \\
83 & 0.19 & 36 \\
$94.9-97.5$ & $0.183-0.089$ & 38 \\
$92-98$ & $0.1-0.6$ & This work \\
$96.1-96.4$ & 0.101 & \\
\hline
\end{tabular}

This light-weight SiCNW sponge generated via sugar-blowing assisted in-situ carbothermal reduction process displayed low density of $115-125 \mathrm{mg} / \mathrm{cm}^{3}$ and high porosity up to $96.4 \%$. Different from most of the $\mathrm{SiC}$ sponges/foams that fail in a brittle manner under compression, this sponge with an exciting core-shell structure containing long and smooth nanowires in the shell layer and relatively short whiskers in the inner part provides a combination of excellent robustness and resilience during cyclic 
compression. The monotonic and cyclic compression tests have revealed that the shell layer comprising highly flexible nanowires contributes to the recoverability (at a $\varepsilon=20 \%$ ) and outstanding brittleness resistance, whilst the inner part ensures the high compressive strength and structural integrity.

Benefiting from the intrinsically stable and flexible structural characteristics, the multifunctional SiCNW sponges offer a variety of unique advantages:

First is its pressure-dependant electromechanical performance. Compared with most currently developed foam-like sensors that focused on finger force detection, the present sensors are capable of large dynamic compressive load measurements in the higher regime of $\sim 130.2-\sim 651 \mathrm{kPa}$. This offers great potential for monitoring vibration, acoustics, and structural damage, or impacts to human body. The present SiCNW sensor also provides large resistance response, ranging from 500 to 1000 $\Omega$, which is 500 times wider than that of carbon-polymer composites-based sensors, ${ }^{9}$ thus offering much higher reliability. Further, unlike carbon-based nanostructures that rely on strong Van der Waals interactions between them, the connections of SiCNWs within the core-shell structure of the sponge not only create good sensing capability but also provide excellent structural robustness and compressibility, since they are less likely to aggregate upon testing. Therefore, the resistance remained constant over many cycles, which means a good damage tolerant and drift-free response.

Additionally, owing to the nanowire constituents and the high sponge porosity, the SiCNW sponges exhibit superior thermal insulation performance to other porous ceramics, with a thermal conductivity of merely $0.101 \mathrm{Wm}^{-1} \mathrm{~K}^{-1}$. This excellent thermal insulation property demonstrates huge potentials for utilisations in harsh environments including high temperatures, corrosive, etc, which are in high demand for applications in thermal energy conversion systems, electronic devices, and heat-resisting materials that can be hardly fulfilled by polymer or carbon-based sponges.

\section{Conclusion}

In summary, we have created lightweight multifunctional SiCNW sponges via the simple sugarblowing assisted carbothermal reduction. The material with unique core-shell macrostructure exhibits a combination of low density, high compressive strength, and recoverability under cyclic compressive loading. Although the shell layer experienced an initial densification under the first compression, the abundant rigid $\mathrm{SiC}$ whiskers in the core part provide high structural robustness. We have demonstrated that the SiCNW sponge as pressure sensor possesses high sensitivity, large resistance response, and excellent stability, being a promising candidate for fabricating electromechanical device by substituting current polymer-carbon composites. Combined with their low thermal 
conductivity and high chemical stability, the SiCNW sponges are envisaged to be highly useful for a series of energy-storage and electronic applications.

\section{Supporting Information}

XRD profiles of $\mathrm{SiC} / \mathrm{C}$ with different starting sugar: $\mathrm{SiO}_{2}$ contents; Weight percentage of $\alpha-\mathrm{SiC}$ and $\beta$-SiC in purified SiC sponges; Digital photos of $\mathrm{SiC} / \mathrm{C}$ and $\mathrm{SiC}$ sponges; FT-IR spectra of SiCNW sponges; SEM images of the as-obtained SiC/C sponges; Comparison of compressive modulus between present SiCNW sponges with other carbon and ceramic nanostructures; Electrical resistance of $\mathrm{SiC} / \mathrm{C}$ sponges under cyclic compression load for 400 cycles. 


\section{References}

(1) Niu, D.; Jiang, W.; Ye, G.; Wang, K.; Yin, L.; Shi, Y.; Chen, B.; Luo, F.; Liu, H. GrapheneElastomer Nanocomposites Based Flexible Piezoresistive Sensors for Strain and Pressure Detection. Mater. Res. Bull. 2018, 102, 92-99.

(2) Aldraihem, O. J.; Akl, W. N.; Baz, A. M. Nanocomposite Functional Paint Sensor for Vibration and Noise Monitoring. Sensors Actuators, A Phys. 2009, 149, 233-240.

(3) Xu, S.; Vogt, D. M.; Hsu, W.-H.; Osborne, J.; Walsh, T.; Foster, J. R.; Sullivan, S. K.; Smith, V. C.; Rousing, A. W.; Goldfield, E. C.; Wood, R. J. Biocompatible Soft Fluidic Strain and Force Sensors for Wearable Devices. Adv. Funct. Mater. 2018, 29, 1807058.

(4) Xu, R.; Zhang, H.; Cai, Y.; Ruan, J.; Qu, K.; Liu, E.; Ni, X.; Lu, M.; Dong, X. Flexible and Wearable 3D Graphene Sensor with $141 \mathrm{KHz}$ Frequency Signal Response Capability. Appl. Phys. Lett. 2017, 111, 103501.

(5) Gong, S.; Schwalb, W.; Wang, Y.; Chen, Y.; Tang, Y.; Si, J.; Shirinzadeh, B.; Cheng, W. A Wearable and Highly Sensitive Pressure Sensor with Ultrathin Gold Nanowires. Nat. Commun. 2014, $5,1-8$.

(6) Rizvi, R.; Cochrane, B.; Biddiss, E.; Naguib, H. Piezoresistance Characterization of Poly(Dimethyl-Siloxane) and Poly(Ethylene) Carbon Nanotube Composites. Smart Mater. Struct. 2011, 20, 094003.

(7) Su, J.-D.; Jia, X.-S.; Li, J.-T.; Lou, T.; Yan, X.; Sun, J.-L.; Chen, J.-H.; Long, Y.-Z. A ThreeDimensional Porous Conducting Polymer Composite with Ultralow Density and Highly Sensitive Pressure Sensing Properties. J. Nanomater. 2016, 2016, 5164012.

(8) Liu, H.; Huang, W.; Gao, J.; Dai, K.; Zheng, G.; Liu, C.; Shen, C.; Yan, X.; Guo, J.; Guo, Z. Piezoresistive Behavior of Porous Carbon Nanotube-Thermoplastic Polyurethane Conductive Nanocomposites with Ultrahigh Compressibility. Appl. Phys. Lett. 2016, 108, 011904.

(9) Boland, C. S.; Khan, U.; Binions, M.; Barwich, S.; Boland, J. B.; Weaire, D.; Coleman, J. N. Graphene-Coated Polymer Foams as Tuneable Impact Sensors. Nanoscale 2018, 10, 5366-5375.

(10) Wang, H.; Zhang, X.; Wang, N.; Li, Y.; Feng, X.; Huang, Y.; Zhao, C.; Liu, Z.; Fang, M.; Ou, G.; Gao. H.; Li, X.; Wu, H. Ultralight, Scalable, and High-Temperature-Resilient Ceramic Nanofiber Sponges. Science Advances 2017, 3, e1603170.

(11) Duong-viet, C.; Ba, H.; El-berrichi, Z.; Nhut, J. Silicon Carbide Foam as a Porous Support Platform. New J. Chem. 2016, 40, 4285-4299.

(12) Shcherban, N. D.; Filonenko, S. M.; Yaremov, P. S.; Sergiienko, S. A.; Ilyin, V. G.; Murzin, D. Y. Carbothermal Synthesis of Porous Silicon Carbide Using Mesoporous Silicas. J. Mater. Sci. 2017, 52, 3917-3926. 
(13) Liang, C.; Wang, Z.; Wu, L.; Zhang, X.; Wang, H.; Wang, Z. Light and Strong Hierarchical Porous SiC Foam for Efficient Electromagnetic Interference Shielding and Thermal Insulation at Elevated Temperatures. ACS Appl. Mater. Interfaces 2017, 9, 29950-29957.

(14) Satori, K.; Kishimoto, H.; Park, J. S.; Jung, H. C.; Lee, Y. J.; Kohyama, A. Thermal Insulator of Porous SiC/SiC Composites for Fusion Blanket System. In IOP Conference Series: Materials Science and Engineering. IOP Publishing. 2011, 18, 162019.

(15) Mélinon, P.; Masenelli, B.; Tournus, F.; Perez, A. Playing with Carbon and Silicon at the Nanoscale. Nat. Mater. 2007, 6, 479-490.

(16) Eom, J.; Kim, Y.; Kim, K. J. Suppression of Free Si Formation during Liquid Phase Sintering of Polysiloxane-Derived, Porous Silicon Carbide Ceramics. Journal of the Ceramic Society of Japan 2010, 118, 102-106.

(17) Chabi, S.; Rocha, V. G.; Garclá-Tunİón, E.; Ferraro, C.; Saiz, E.; Xia, Y.; Zhu, Y. Ultralight, Strong, Three-Dimensional SiC Structures. ACS Nano 2016, 10, 1871-1876.

(18) Chabi, S.; Chang, H.; Xia, Y.; Zhu, Y. From Graphene to Silicon Carbide : Ultrathin Silicon Carbide Flakes. Nanotechnology 2016, 27, 75602.

(19) Shcherban, N. D. Review on Synthesis, Structure, Physical and Chemical Properties and Functional Characteristics of Porous Silicon Carbide. J. Ind. Eng. Chem. 2017, 50, 15-28.

(20) Hu, C.; Hong, W.; Xu, X.; Tang, S.; Du, S.; Cheng, H.-M. Sandwich-Structured C/C-SiC Composites Fabricated by Electromagnetic-Coupling Chemical Vapor Infiltration. Sci. Rep. 2017, 7, 13120.

(21) Cheng, C.; Li, H.; Fu, Q.; Li, L.; Guo, L.; Yin, X.; Tian, X. Effects of Pyrocarbon on Morphology Stability of SiC Nanowires at High Temperatures. J. Am. Ceram. Soc. 2018, 101, 36943702.

(22) Vanhaecke, E.; Ivanova, S.; Deneuve, A.; Ersen, O.; Edouard, D.; Winé, G.; Nguyen, P.; Pham, C.; Pham-Huu, C. 1D SiC Decoration of SiC Macroscopic Shapes for Filtration Devices. J. Mater. Chem. 2008, 18, 4654.

(23) Li, Z.; Chen, B.; Wang, X.; Nie, J.; Ma, G. Electrospun Bamboo-like Fe3C Encapsulated FeSi-N Co-Doped Nanofibers for Efficient Oxygen Reduction. J. Colloid Interface Sci. 2019, 546, 231239.

(24) Niu, Q.; Guo, J.; Chen, B.; Nie, J.; Guo, X.; Ma, G. Bimetal-Organic Frameworks/Polymer Core-Shell Nanofibers Derived Heteroatom-Doped Carbon Materials as Electrocatalysts for Oxygen Reduction Reaction. Carbon 2017, 114, 250-260.

(25) Wang, B.; Sun, L.; Wu, N.; Wang, Y. Combined Synthesis of Aligned SiC Nanofibers via Electrospinning and Carbothermal Reduction. Ceram. Int. 2017, 43, 10619-10623. 
(26) Si, Y.; Yu, J.; Tang, X.; Ge, J.; Ding, B. Ultralight Nanofibre-Assembled Cellular Aerogels with Superelasticity and Multifunctionality. Nat. Commun. 2014, 5, 1-9.

(27) Wang, X.; Zhang, Y.; Zhi, C.; Wang, X.; Tang, D.; Xu, Y.; Weng, Q.; Jiang, X.; Mitome, M.; Golberg, D.; Bando, Y. Three-Dimensional Strutted Graphene Grown by Substrate-Free Sugar Blowing for High-Power-Density Supercapacitors. Nat. Commun. 2013, 4, 2905.

(28) Wang, X.-B.; Jiang, X.-F.; Bando, Y. Blowing Route towards Advanced Inorganic Foams. Bull. Chem. Soc. Jpn. 2019, 92, 245-263.

(29) Shen, D.; Zhan, Z.; Liu, Z.; Cao, Y.; Zhou, L.; Liu, Y.; Dai, W.; Nishimura, K.; Li, C.; Lin, C. -T; Jiang, N; Yu, J. Enhanced Thermal Conductivity of Epoxy Composites Filled with Silicon Carbide Nanowires. Sci. Rep. 2017, 7, 1-11.

(30) Chen, J.; Ding, L.; Xin, L.; Zeng, F.; Chen, J. Thermochemistry and Growth Mechanism of SiC Nanowires. J. Solid State Chem. 2017, 253, 282-286.

(31) Su, L.; Wang, H.; Niu, M.; Fan, X.; Ma, M.; Shi, Z.; Guo, S.-W. Ultralight, Recoverable, and High-Temperature-Resistant SiC Nanowire Aerogel. ACS Nano 2018, 12, 3103-3111.

(32) Slack, G. A. Thermal Conductivity of Pure and Impure Silicon, Silicon Carbide, and Diamond. J. Appl. Phys. 1964, 35, 3460-3466.

(33) Živcová, Z.; Gregorová, E.; Pabst, W.; Smith, D. S.; Michot, A.; Poulier, C. Thermal Conductivity of Porous Alumina Ceramics Prepared Using Starch as a Pore-Forming Agent. J. Eur. Ceram. Soc. 2009, 29, 347-353.

(34) Liu, C. Y.; Tuan, W. H.; Chen, S. C. Preparation of Porous SiC Ceramics for Thermal Dissipation Purposes. Ceram. Int. 2015, 41, 4564-4568.

(35) Eom, J. H.; Kim, Y. W.; Song, I. H.; Kim, H. D. Processing and Properties of PolysiloxaneDerived Porous Silicon Carbide Ceramics Using Hollow Microspheres as Templates. J. Eur. Ceram. Soc. 2008, 28, 1029-1035.

(36) Wang, Y.; Chen, Z.; Jiang, Y.; Yu, S.; Xu, T.; Li, B.; Chen, Z. Densification Control and Analysis of Outer Shell of New High-Temperature Vacuum Insulated Composite. Mater. Res. Express. 2017, 4, 115601.

(37) Vijayan, S.; Wilson, P.; Sreeja, R.; Prabhakaran, K. Ultralight SiC Foams with Improved Strength from Sucrose and Silicon Powder Using Magnesium Nitrate Blowing Agent. Mater. Lett. 2017, 194, 126-129.

(38) Ferraro, C.; Garcia-Tuñon, E.; Rocha, V. G.; Barg, S.; Fariñas, M. D.; Alvarez-Arenas, T. E. G.; Sernicola, G.; Giuliani, F.; Saiz, E. Light and Strong SiC Networks. Adv. Funct. Mater. 2016, 26, $1636-1645$. 